\title{
Chronic Lymphocytic Leukaemia/ Small-Cell Lymphocytic Lymphoma of the Lacrimal Sac: A Case Series
}

\author{
Yamini Krishna $^{a}$ Luciane D. Irion $^{d}$ Sozan Karim $^{b}$ Aruna Dharmsena ${ }^{\mathrm{e}}$ \\ Austin McCormick ${ }^{c}$ Sarah E. Coupland ${ }^{a, b}$ \\ ${ }^{a}$ Department of Cellular Pathology, Royal Liverpool and Broadgreen University Hospital Trust, ${ }^{b}$ Department of \\ Molecular and Clinical Cancer Medicine, University of Liverpool, and ' Department of Ophthalmology, Aintree \\ University Hospital, Liverpool, and Departments of ${ }^{\mathrm{d}} \mathrm{Histopathology}$ and ${ }^{\mathrm{e}}$ Ophthalmology, Central Manchester \\ University Hospitals NHS Foundation Trust, Manchester, UK
}

\section{Established Facts}

- Lymphomas of the lacrimal sac are very rare, accounting for $~ 2-6 \%$ of lacrimal sac malignancies, which tend to be of epithelial origin.

- Lacrimal sac lymphomas may represent secondary disease of systemic lymphoma (e.g., chronic lymphocytic leukaemia/small-cell lymphocytic lymphoma) and should be suspected in such patients with persistent nasolacrimal obstruction.

\section{Novel Insights}

- Small nasolacrimal sac biopsies can yield sufficient and suitable DNA, even in the fixed state for nextgeneration sequencing and assessment of somatic mutation status, relevant for prognostication.

- Although involvement of the lacrimal sac by chronic lymphocytic leukaemia/small-cell lymphocytic lymphoma is usually only seen in advanced-stage disease, the clinical course of these patients after diagnosis is variable.

\section{Keywords}

Lacrimal sac · Lymphoma · Chronic lymphocytic leukaemia • Dacryocystitis · Epiphora

\section{Abstract \\ Background: Lymphomas of the lacrimal sac are rare, ac- counting for less than $10 \%$ of lacrimal sac malignant tu-}

mours. They may present with symptoms typical of secondary acquired nasolacrimal duct obstruction and are thus often misdiagnosed. Methods: Case series and literature review. Results: Herein we describe 3 cases of chronic lymphocytic leukaemia (CLL)/small-cell lymphocytic lymphoma (SLL) of the lacrimal sac with immunohistochemical and in 1 case molecular confirmation. Conclusion: Lymphomas of the lacrimal sac should be suspected in patients with known

\section{KARGER}

(C) 2017 S. Karger AG, Basel

E-Mail karger@karger.com

www.karger.com/oop
Prof. Sarah E. Coupland

Department of Molecular and Clinical Cancer Medicine

University of Liverpool, William Henry Duncan Building

6 West Derby Street, Liverpool L7 8TX (UK)

E-Mail s.e.coupland@ liverpool.ac.uk 
CLL presenting with epiphora and dacryocystitis. During dacryocystorhinostomy, an incisional biopsy of the lacrimal sac is essential for confirming CLL/SLL involvement and may guide treatment.

(c) 2017 S. Karger AG, Basel

Malignant tumours of the lacrimal sac are uncommon, with close to $90 \%$ of these being of epithelial origin. Lymphomas of the lacrimal sac are rare [1-3]. They may present with symptoms typical of secondary acquired nasolacrimal obstruction including epiphora with a medial canthal mass and are thus often misdiagnosed. It should be suspected in patients with known chronic lymphocytic leukaemia (CLL) presenting with epiphora and dacryocystitis. During dacryocystorhinostomy (DCR), an incisional biopsy of the lacrimal sac is essential for confirming CLL/small-cell lymphocytic lymphoma (SLL) involvement and may therefore guide adjuvant treatment [1-7].

All patients had signed a written informed consent form and the study was conducted in accordance with the Declaration of Helsinki.

\section{Case Reports}

Case 1

An 89-year-old male with past medical history of CLL and left external DCR presented to the eye department with a painful, discharging, cystic swelling in the right lacrimal sac area. He was treated with antibiotics for his right-sided dacryocystitis, and later listed for a right external DCR. Perioperatively, the lacrimal sac was noted to be inflamed, and hence an incisional biopsy was taken.

The specimen measured $8 \times 5 \times 3 \mathrm{~mm}$. Histological examination revealed a diffuse atypical lymphocytic infiltrate with mild nodularity, intermediate-sized cells with coarse nucleus and prominent nucleoli, and large cells with prominent nucleoli in proliferation centres (Fig. 1a, b). Ki-67 immunostaining showed a moderate growth fraction with mitotic cells localized in proliferation centres (Fig. 1c). Further evaluation with immunohistochemistry (IHC) confirmed monotypical B cells with positive staining for CD5, CD20, CD23, CD79a (Fig. 1d-f), and BCL-2 with synthesis of $\operatorname{IgM}, \operatorname{IgD}$, and equivalent staining of kappa and lambda light-chains, with a moderate background scattering of CD3+ T cells. The following immunostains were negative: cyclinD1, CD21, CD10, and BCL-6. Histological and IHC features were consistent with diffuse, well-differentiated CLL/SLL. Immunoglobulin heavy-chain polymerase chain reaction demonstrated a monoclonal B-cell population. Furthermore, next-generation sequencing was performed as previously described [8]. Briefly, target exons of 15 selected genes relevant to CLL were sequenced using Ion Torrent Personal Genome Machine and Torrent Suite Pipeline v4 (Life Technologies, UK). Genes included were: TP53 (exons 2-11) and SF3B1 (exons 8-20), ATM (exons 2-63), MYD88 (exons 2-5), BIRC3 (exons 2-9), NOTCH1 (exon 34), XPO1 (exons 11-16), LRP1B (exons 34-86), FBXW7 (exons 5-12), HIST1H1E (exon 1), ZFPM2 (exon 8), SAMHD1 (exons 1-16), CHD2 (exons 12-36), POT1 (exons 5-19), and PCLO (exons 2-24). This molecular panel has previously been reported to be associated with extensive nodal involvement indicating advanced stages of CLL and thus relevant for its prognosis [8]. Nextgeneration sequencing was performed in this case to ascertain whether or not lacrimal sac involvement is associated with any of these gene mutations. No somatic gene mutations were detected in the sample.

The patient was already under the care of haematologists and had received chemotherapy - previous course of chlorambucil followed by bendamustine. He was given a further single lower dose of bendamustine. His lacrimal stents were removed 6 months after successful DCR and he was given a further 6-month follow-up appointment. Prior to his appointment at the eye department, the patient had surgery for a sebaceous cell carcinoma on his calf involving excision and grafting, which unfortunately failed and was found to be infected with Pseudomonas. He subsequently developed sepsis and was treated with intravenous antibiotics but rapidly deteriorated and died within 4 days of admission.

Case 2

A 66-year-old male with known CLL in the past presented with a 6-month history of epiphora and a painless hard mass over the right lacrimal sac. Computed tomography revealed a soft tissue mass, measuring approximately $20 \times 22 \times 16 \mathrm{~mm}$, in the medial canthal region extending into the lacrimal fossa and nasolacrimal duct into the nasal cavity. A right external DCR with an incisional biopsy of the lacrimal sac was performed.

Histopathological analysis showed a nodular and diffuse infiltration by intermediate-sized, round lymphocytes punctuated by pale-staining proliferation centres containing pro-lymphocytes and para-immunoblasts. There was an unusual degree of stromal fibrosis and marked perineural and perivascular sclerosis. Tumour cells had a classical CLL phenotype: CD5+, CD20+, CD21+, CD23+, CD79a+, MUM-1+, and BCL-2+ with synthesis of IgM. CD3, CD10, cyclin-D1, and BCL-6 stains were negative. The Ki67 growth fraction was $<5 \%$. Plasma cells within sclerotic areas showed indeterminate light-chain staining. Overall, features were consistent with lacrimal sac involvement by CLL/SLL.

The patient had low-dose local radiation of the lacrimal sac area alone, as further disease was not detected elsewhere on staging. The patient was re-referred to the eye department 2 years later for persistent epiphora secondary to post-irradiation scarring. There was a complete response locally, but the patient suffered a relapse of the CLL elsewhere, received further chemotherapy, and was subsequently lost to follow-up at the eye department.

\section{Case 3}

A 75-year-old female with a past medical history of CLL (p53 deletion) treated with fludarabine and cyclophosphamide and successful left external DCR for recurrent dacryocystitis but local drainage of the right lacrimal sac due to relapse of her CLL, presented with a recent 6-month history of intermittent right eye epiphora. Clinically there was right nasolacrimal duct obstruc- 

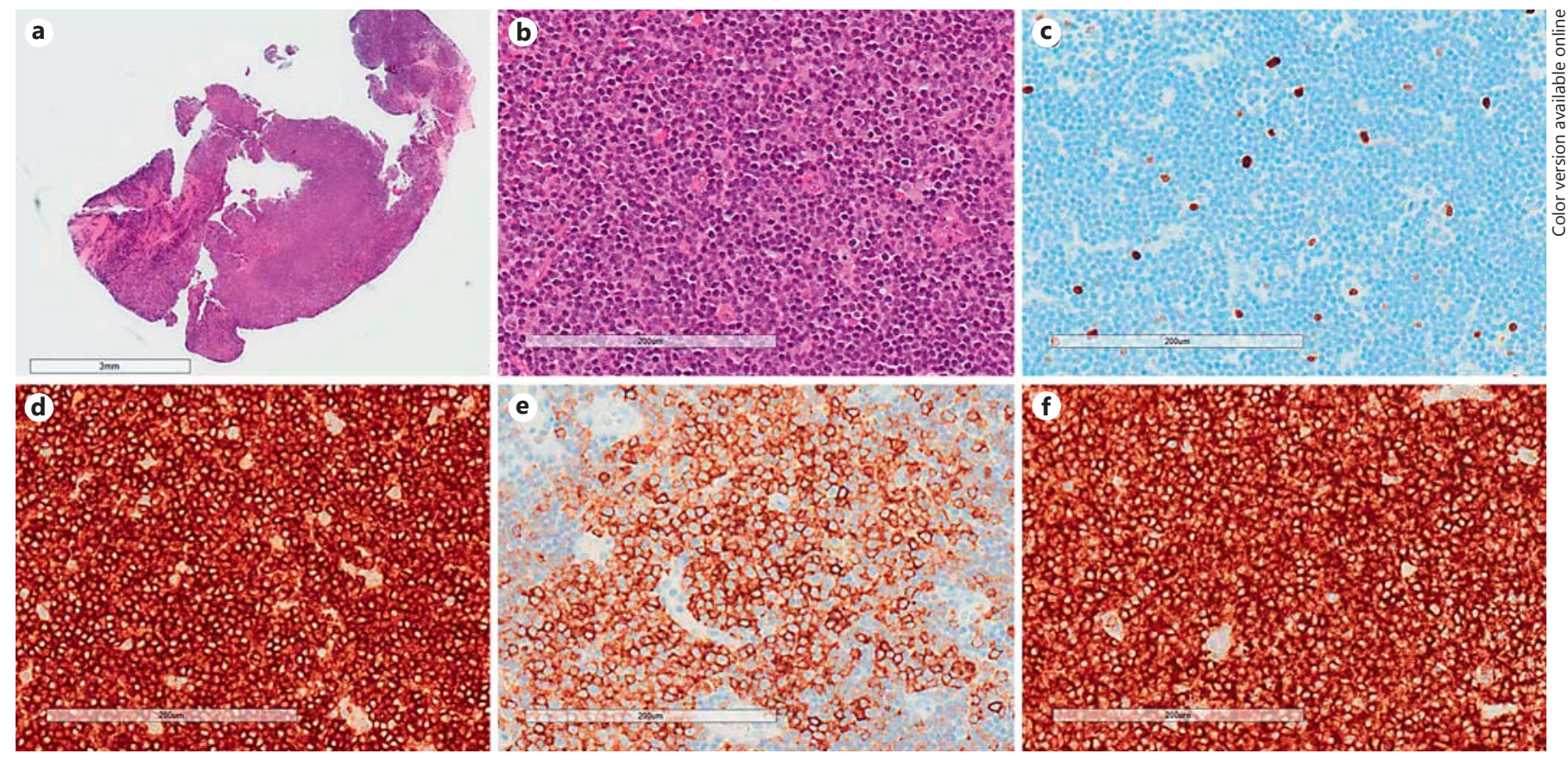

Fig. 1. Histological examination of lacrimal sac specimen. a Lacrimal sac biopsy (H\&E). b Diffuse lymphocytic infiltrate showing intermediate-sized cells with prominent nucleoli $(\mathrm{H} \& \mathrm{E}, \times 20)$. c Ki-67 immunostaining showing a moderate proliferation ratio. d-f Immunohistochemistry $\left(3,3^{\prime}\right.$-diaminobenzidine $\left.[D A B], \times 20\right)$ showing $B$ cells with positive staining for CD5 (d), CD23 (e), and CD20 (f).
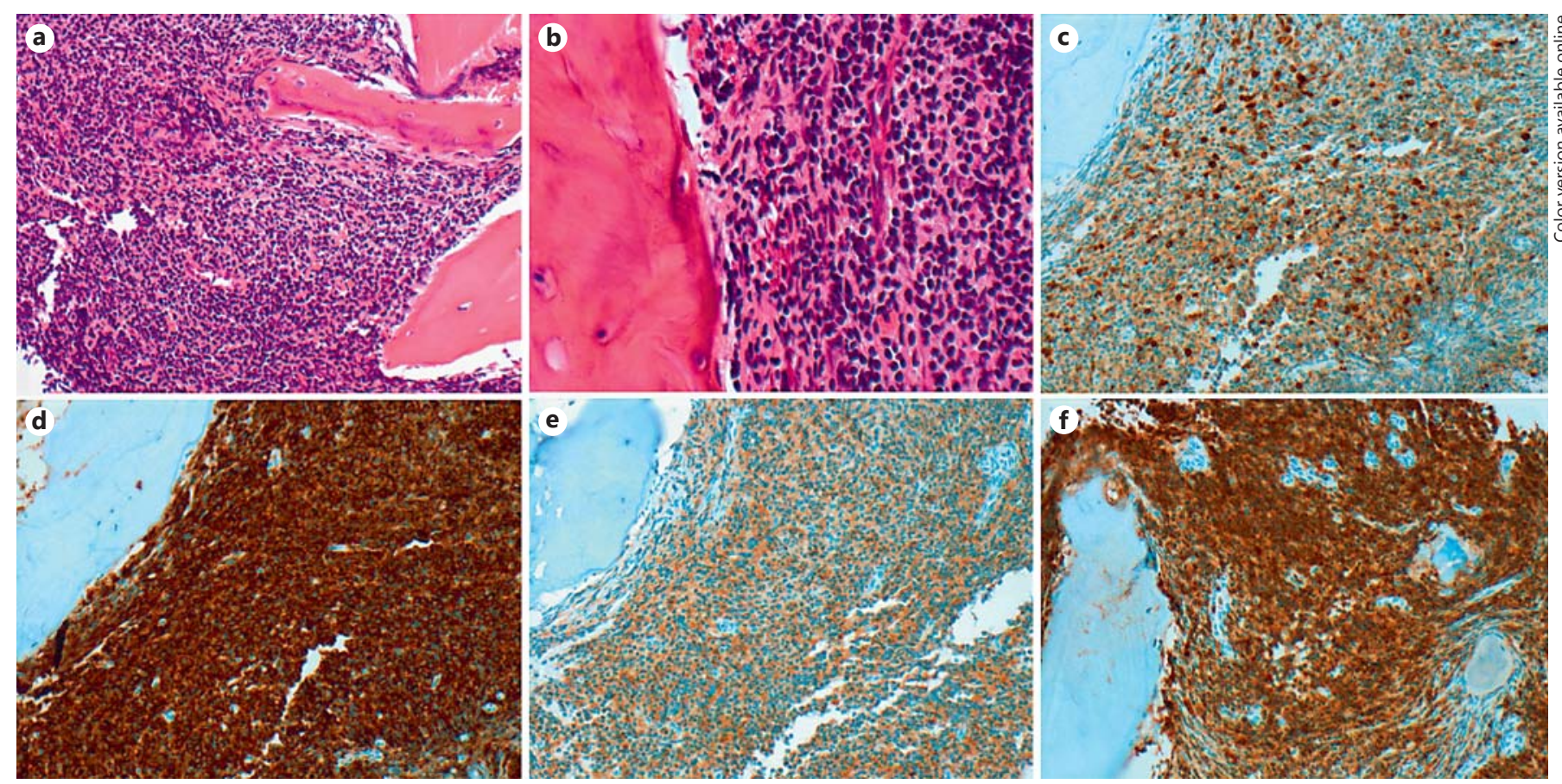

Fig. 2. Histological assessment of frontal bone biopsy. a, b Dense infiltration of intratrabecular stroma by lymphoma (a H\&E, $\times 10$ and $\mathbf{b} H \& E, \times 20)$. c-f Immunostaining (DAB, $\times 10)$ showing positive staining for CD5 (c), CD20 (d), CD23 (e), and CD79a (f).

Ocul Oncol Pathol 2017;3:224-228

DOI: $10.1159 / 000455148$
Krishna/Irion/Karim/Dharmsena/ McCormick/Coupland 
tion but no palpable mass. During external DCR, the right nasal process of frontal bone was noted to have a soft consistency with a spongy mucoid appearance and therefore prompted a biopsy. There was no obvious tumour noted in the lacrimal sac.

Histological assessment was limited due to a very small specimen and marked traction artefact. Of note, however, small fragments of trabecular bone showed dense infiltration of the intratrabecular stroma by lymphoma (Fig. 2a, b). The tumour comprised small lymphocytes with scanty pale cytoplasm and small, round nuclei with condensed chromatin and no nucleoli. The cell infiltrate was CD20+, CD79a+, CD5+, CD23+ (Fig. 2c-e), BCL$2+, \operatorname{IgM}+$, and IgD+ with kappa light-chain restriction, but negative for CD10, p53, MUM-1, CD43, and cyclin D1. The Ki-67 growth fraction was $<5 \%$. There was marked reduction of $\mathrm{CD} 3+$ and $\mathrm{CD} 5+$ reactive $\mathrm{T}$ cells. Histological and $\mathrm{IHC}$ appearances were consistent with involvement by CLL/SLL.

The patient was referred back to haematologists and treated with chlorambucil and ofatumumab. She has been in remission for 5 years.

\section{Discussion}

Lymphomatous involvement of the lacrimal sac may be primary or secondary. There are $<70$ primary cases and few case reports/series of secondary involvement. Previous reporting would have been variable due to lack of global consensus regarding lymphoma classifications until the Revised European-American Classification of Lymphoid Neoplasms, the WHO classification, and later the EORTC ophthalmic oncology task force study defined the clinical and histopathological characteristics [1$3,9-10]$. From the EORTC study of 15 primary lacrimal sac lymphoma cases, $33 \%$ of cases were classified as diffuse large B cell lymphoma (DLBCL; non-Hodgkin's lymphoma), 33\% as extranodal marginal zone B cell lymphoma of mucosa-associated lymphoid tissue (MALT lymphoma), $20 \%$ as transitional MALT lymphoma with features between MALT lymphoma and DLBCL, and $13 \%$ as unclassified B cell lymphomas [10]. Of the other in the literature, it appears that DLBCL and MALT lymphomas occur with approximately equal frequency $[1-3$, $5,7,9-11]$. However, DLBCL has been more frequently described in association with systemic lymphoma $[2-3$, 7].

Lymphomas account for $\sim 2-6 \%$ of lacrimal sac malignant tumours, present in generally older patients with symptoms typical of secondary acquired nasolacrimal obstruction including epiphora and medial canthal mass, and are thus often misdiagnosed as acute or chronic dacryocystitis [4-7]. It should be suspected in patients with known CLL presenting with epiphora and dacryocystitis. DCR with stenting is well tolerated and effective at allevi- ating symptoms. During DCR, an incisional biopsy of the lacrimal sac is essential for confirming CLL/SLL involvement, even in the absence of obvious swelling (as in 1 of our cases), and may guide adjuvant chemo- and/or radiotherapy treatment $[1-4,6]$. Treatment is usually a combination of surgery, irradiation, and/or chemotherapy (notably regimens involving chlorambucil), but no commonly agreed treatment regimen for periocular lymphoma exists because of the limited number of patients seen $[2-3,7,10,11]$.

In keeping with earlier reports [2,3], our patients had systemic CLL with secondary lacrimal sac infiltration and were elderly, but one had presumed bilateral involvement (2 DCRs) and one had bony destruction, which are rare. Two of the patients had been treated with chemotherapy (including chlorambucil), whilst one had low-dose local area irradiation as no further disease was detected.

In summary, CLL/SLL infiltration of the lacrimal sac is rare and should be suspected in any patient with systemic CLL presenting with epiphora and/or lacrimal sac area mass. Herein we present 3 cases with immunohistochemical confirmation and one where molecular testing was available - which, to our knowledge, has not been previously reported. However, we do recognize that no somatic mutations were detected even though this molecular panel has previously been reported to be relevant for CLL prognosis and treatment response [8]. Whether or not lacrimal sac involvement is associated with the same somatic gene mutations requires further evaluation in a larger case series.

\section{Statement of Ethics}

Informed consent had been obtained and care has been taken to preserve the patients' anonymity. The authors state that their study complies with the Declaration of Helsinki.

\section{Disclosure Statement}

The authors declare no conflict of interest. 


\section{References}

1 Gao HW, Lee HS, Lin YS, Sheu LF: Primary lymphoma of nasolacrimal drainage system: a case report and literature review. Am J Otolaryngol 2005;26:356-359.

2 Litschel R, Siano M, Tasman AJ, Cogliatti S: Nasolacrimal duct obstruction caused by lymphoproliferative infiltration in the course of chronic lymphocytic leukemia. Allergy Rhinol Providence 2015;6:191-194.

3 Yip CC, Bartley GB, Habermann TM, Garrity JA: Involvement of the lacrimal drainage system by leukemia or lymphoma. Ophthal Plast Reconstr Surg 2002;18:242-246.

4 Benger RS, Frueh BR: Lacrimal drainage obstruction from lacrimal sac infiltration by lymphocytic neoplasia. Am J Ophthalmol 1986;101:242-245.
5 Ferry JA, Fung CY, Zukerberg L, Lucarelli MJ, Hasserjian RP, Preffer FI, Harris NL: Lymphoma of the ocular adnexa: a study of 353 cases. Am J Surg Path 2007;31:170-184.

6 Karesh JW, Perman KI, Rodrigues MM: Dacryocystitis associated with malignant lymphoma of the lacrimal sac. Ophthalmology 1993; 100:669-673.

7 Montalban A, Liétin B, Louvrier C, Russier M, Kemeny JL, Mom T, Gilain L: Malignant lacrimal sac tumors. Eur Ann Otorhinolaryngol Head Neck Dis 2010;127:165-172.

8 Karim S, Johnson G, Pettitt A, Lin K: A longitudinal study of next-generation sequencing in chronic lymphocytic leukaemia using Ion Torrent PGM shows evidence of chemotherapy-induced mutagenesis and a snowballing effect due to loss of p53/ATM-mediated DNA repair. Haematologica 2015;100:222-223.
9 Auw-Haedrich C, Coupland SE, Kapp A Schmitt-Gräff A, Buchen R, Witschel H: Long term outcome of ocular adnexal lymphoma subtyped according to the REAL classification. Br J Ophthalmol 2001;85:63-69.

10 Sjö L, Ralfkiaer E, Juhl BR, Prause JU, Kivelä T, Auw-Haedrich C, Bacin F, Carrera M, Coupland SE, Delbosc B, Ducrey N, Kantelip B, Kemeny JL, Meyer P, Sjö NC, Heegaard S: Primary lymphoma of the lacrimal sac: an EORTC ophthalmic oncology task force study. Br J Ophthalmol 2006;90:1004-1009.

11 Parmar DN, Rose GE: Management of lacrimal sac tumours. Eye 2003;17:599-606. 